\title{
Experimental Investigation on Rotordynamic Characteristics and Rotor System Stability of a Novel Negative Dislocated Seal
}

\author{
Dan Sun, ${ }^{1}$ Xudong Wang, ${ }^{2}$ Chengwei Fei $\mathbb{D}^{3},{ }^{3}$ Huan Zhao, ${ }^{1}$ Guochen Zhang, \\ and Wenzhong Tang ${ }^{4}$ \\ ${ }^{1}$ Liaoning Key Lab of Advanced Test Technology for Aerospace Propulsion System, Shenyang Aerospace University, \\ Shenyang 110136, China \\ ${ }^{2}$ AECC Sichuan Gas Turbine Establishment, Chengdu 610500, China \\ ${ }^{3}$ Department of Aeronautics and Astronautics, Fudan University, Shanghai 200433, China \\ ${ }^{4}$ School of Computer Science and Engineering, Beihang University, Beijing 100191, China \\ Correspondence should be addressed to Chengwei Fei; cwfei@fudan.edu.cn
}

Received 12 January 2019; Accepted 29 May 2019; Published 17 June 2019

Academic Editor: Luca Landi

Copyright ( 2019 Dan Sun et al. This is an open access article distributed under the Creative Commons Attribution License, which permits unrestricted use, distribution, and reproduction in any medium, provided the original work is properly cited.

\begin{abstract}
Air-induced force generated in seals is one key factor on the stability of the rotor system. In this paper, a novel negative dislocated seal (NDS) was developed in respect of dislocated bearing theory, to reduce hydrodynamic pressure effect and air-induced force and improve rotor stability as well. A test rig was built to test rotordynamic characteristics and rotor stability of the NDS. The rotordynamic characteristics of seals were investigated based on the unbalanced synchronous excitation method, and seal-rotor system stability was evaluated by the identification method with an electromagnetic bearing exciter. The effects of both rotating speed and inlet/outlet pressure ratio on the rotordynamic characteristics and rotor stability of both NDS and conventional cylindrical labyrinth seal were experimentally investigated. The results show that with the increasing rotating speed, inlet/outlet pressure ratio is promising to reduce the direct stiffness coefficients of seals and the logarithmic decrement rate of seal-rotor system and enhance both cross stiffness and damping coefficient as well. Besides, the developed NDS effectively reduces crossstiffness coefficients and increases direct damping coefficients and the logarithmic decrement rate of the seal-rotor system, relative to the conventional cylindrical seal. The proposed seal can effectively improve seal stability of turbomachinery.
\end{abstract}

\section{Introduction}

For turbomachinery such as steam turbine, centrifugal compressor, and aeroengine, seals are one critical component and play the key role of preventing working medium leakage, reducing energy consumption, and improving work efficiency [1-3]. Besides, seals also generate a prominent flow-induced force. The force severally influences rotor vibration for high-parameter turbomachinery. The high parameters indicate high temperature, high pressure, and high rotation speed for turbomachinery. With the increasing improvement of working medium parameters, it is inevitable for the seal flow-induced vibration to lead to the rotor instability of turbomachinery.

To reduce flow-induced force and improve the stability of seals, numerous seals have been designed in respect of different structures, which are regarded as two types of both anti-preswirl seal and damping seal. As for anti-preswirl seal, the anti-preswirl idea was developed by Muszynska in the 1980s [4]. The anti-preswirl seal can be divided into swirl brake seal and antiswirl seal. The swirl brake seal is commonly installed at the sealed inlet, to stop vortex and resistance to swirl [5]. Sun et al. [6] found that the swirl brake seal is potential to improve the seal stability. Nevertheless, antiswirl seal is to offset the impact of rotor rotation on circumferential airflow by injecting a stream of air with reverse direction to rotor rotation $[7,8]$. Shunt injection is revealed an effective approach to improve the rotordynamic characteristics of labyrinth seal [9-11]. Therefore, the stability of rotor system is commonly disturbed by inappropriate anti-prewhirl seal structure. Some investigations showed that setting anti-prewhirl structure has a certain 
effect, but it has a difficult design and complicated structure and may lead to instability of the rotor system [12]. The concept of damping seal was first proposed by Pragenau in 1982 [13]. The damping seal can improve seal rotordynamic characteristics by increasing the surface roughness of seal structure. Relative to the labyrinth seal, the stator surface of this seal has larger roughness and bigger damping coefficient, which contribute to reduce circumferential flow velocity in the seal, decrease seal cross stiffness, and improve rotor system stability. At present, existing damping seals include honeycomb seal $[14,15]$, hole-pattern seal [16], and pocket damper seal [17].

From the above discussion, it is promising to improve seal stability by changing seal structure and obtain some effect from the angle of the circumferential flow velocity of the seal fluid. In essence, the seal is a circular seal structure although different seals have different mechanisms. With the rotating-machine working increasing medium parameters for cylindrical seal, the hydrodynamic pressure effect, produced by the fluid in circular wedge gap under the condition of the eccentric rotor, enlarges and thus forms more prominent airflow-exciting force [18].

The experimental method of seal rotordynamic characteristics includes time-domain method and frequencydomain method [19]. With respect to the time-domain method, Kwanka et al. discussed the rotordynamic characteristics of the seal gap through testing dynamic pressure in the seal chamber [20]. The time-domain method is affected by pressure measurement precision and measuring point arrangement so that the accuracy based on this method is unacceptable. In comparison, the frequencydomain method has higher accuracy and signal-to-noise ratio $(\mathrm{S} / \mathrm{N})$ [21]. Childs et al. developed a frequencydomain identification model to conduct a large number of investigations on the rotordynamic characteristics of different seals [22-26]. As for the study on seal rotordynamic characteristics with the frequency-domain method, the single-point excitation method is applied, by the excitation at the center of cylinder to acquire vibration response and then obtain impedance function. With regard to the impedance function and the vibration, the rotordynamic coefficients of the seal can be identified. However, this method requires that the movement of the cylinder should be translational. In this case, the model fails very easily when the cylinder is skewing. In line with the engineering experience, the skewing phenomenon is so ubiquitous that the error of deflection has to be considered. Sun et al. [6] proposed a new identification method of seal rotordynamic characteristics based on common-frequency excitation, by extending the single-point excitation model to multipoint excitation and introducing the rotor dynamic unbalance force identification method to evaluate the airflow force under cylinder deflection.

Logarithmic decrement rate is the main parameter of evaluating the stability of rotating machinery rotor [27]. The logarithmic decrement rate can be acquired by calculating the damping ratio of the rotor system. The methods of identifying the damping ratio of the rotor system also include time-domain method and frequency-domain method.
The basic principle of time-domain method is to exert an excitation force on the rotor to make the rotor vibration attenuate freely, then to measure the time-domain data of rotor vibration in the attenuation process, and lastly to compute the logarithmic attenuation rate [28]. Zhong [29] and Cloud [30] employed this method to identify the damping ratio of the rotor system. However, this method is very sensitive to $\mathrm{S} / \mathrm{N}$ and has poor recognition accuracy. The frequency-domain method is used to obtain the frequency response function by excitation force and rotor vibration generated and to analyze the logarithmic decrement rate according to the frequency response function of the tested rotor system. The classical frequency-domain method is halfpower method. Nevertheless, the method is sensitive to noise so that the recognition precision is unacceptable [31]. Lee [32] introduced a complex number to describe the motion status of the rotor, used the direct frequency response function method to identify parameter, and separated positive and negative frequency precessions. Takahashi et al. [33] compared traditional frequency response function recognition with direct frequency response function recognition in the complex field and found that the advantages of recognition with complex field. Although the positive and negative precession can be effectively separated, the model of this method is relatively simple and is only suitable for the occurrence of a single peak in the resonance region. Instead, it is invalid for rotor system parameters identification with multiple peaks in the resonant region under the small damping. To address this issue, Wang et al. [34] proposed the rotor stability identification method based on the electromagnetic bearing exciter. This method effectively improves the recognition accuracy by synthesizing the advantages of both the traditional frequency response function and the complex field direct frequency response function.

Catering for the problem of the increasing hydrodynamic pressure effect of sealed fluid under high parameters and the increase of airflow exciting force, this paper proposes a negative dislocated seal (NDS) structure by considering the dislocated bearing theory. In contrast to the improvement of hydrodynamic pressure effects with the positive-dislocated bearings, a divergent flow field gap can form in the circumferential wedge gap of the NDS, while the conditions to form hydrodynamic pressure effects do not exist, and thus, the seal airflow force is reduced. In this paper, both the rotordynamic characteristics and rotor stability of the NDS and the traditional circular seal are experimentally studied by using seal rotordynamic characteristic identification methods based on unbalanced common-frequency excitation and rotor stability experiment identification method based on the electromagnetic bearing exciter. The influences of dislocated rate, rotational speed, and pressure ratio on seal rotordynamic coefficients and rotor stability are also evaluated.

The remainder of this paper is organized as follows. The NDS mechanism is discussed in Section 2. Section 3 investigates the experiment of seal rotordynamic characteristics including the test rig and identification method of seal rotordynamic characteristics. The identification method includes the equivalent seal force identification model and rotordynamic coefficients identification model. In Section 4, 
the experiment of seal-rotor system stability is conducted by using the test rig and identification method of seal-rotor system stability. The identification method contains the dynamic model of rotor-seal system, the stability evaluation of rotor system with standard-logarithmic decrement rate, and the analysis of the complex field model. Section 5 shows the experimental results and discussions on this study from both seal rotordynamic characteristics and seal stability analyses. Section 6 summarizes some conclusions of this study.

\section{Negative Dislocated Seal}

In view of experimental investigations and field troubleshooting experiences, tangential force induced by labyrinth seals is the major factor inducing load-dependent instability vibration [35]. Seal tangential force results from asymmetrical circumferential pressure distribution under the condition of eccentric rotor. Seals can form hydrodynamic pressure effect when viscous fluid flows from large terminal to small terminal in wedge gap.

To enhance the hydrodynamic pressure effect of journal bearings, dislocated journal bearing is used in rotating machinery. Positive dislocated bearing is to enforce fluid to flow from large terminal to small terminal in wedge gap, to strengthen hydrodynamic pressure effect. The hydrodynamic pressure effect is shown in Figure 1. In Figure 1, $O$ and $O^{\prime}$ are seal center and rotor center, respectively, and $O_{1}$ and $\mathrm{O}_{2}$ indicate the stator centers of dislocated seals, respectively. The structure parameters of cylinder seal and dislocated seal include seal radius $R$, rotor radius $r$, distance $\delta$ between stator center and seal center for dislocated seal, maximum clearance $h_{\max }$ of cylinder seal, minimum clearance $h_{\min }$ of cylinder seal, and rotational speed $\omega$.

As shown in Figure 1, the traditional cylinder seal can form hydrodynamic pressure effect in the area of convergent air wedge under the condition of eccentric rotor, rather than the area of divergent air wedge. Dislocated seal consists of positive dislocated seal and NDS. The fluid in positive dislocated seal flows from large terminal to small terminal in the seal wedge gap, to form intensive hydrodynamic pressure effect. The NDS can form two divergent wedge gaps. Therefore, the NDS can suppress seal hydrodynamic pressure effect to improve the stability of rotor system.

\section{Experiment of Seal Rotordynamic Characteristics}

Figure 2 shows the effect of eccentricity on the leakage characteristics of the new negative dislocation seal and the traditional circular seal. As can be seen from the figure, with the increase of eccentricity, the leakage of the new negative dislocation seal and the traditional circular seal increases. Under the same eccentricity, the leakage of the new negative dislocation seal is larger than that of the traditional circular seal, and with the increase of the dislocation rate, the leakage volume increases, which is mainly due to the dislocation structure of the new negative dislocation seal which increases the air flow through the leakage area, thus increasing the seal leakage.

\section{Experiment of Seal Leakage Characteristics}

4.1. Test Rig of Measuring Seal Rotordynamic Characteristics. Test rig applied to simulate seal rotordynamic characteristics is shown in Figure 3. As for this test rig, the length of rotor is $1,800 \mathrm{~mm}$, the diameter of rotor is $180 \mathrm{~mm}$, the maximum speed is $6,000 \mathrm{rpm}$, and the seal clearance is $0.2 \mathrm{~mm}$. The cylinder of the test rig is tightened by horizontal, vertical, and axial springs. One balancing disc is installed on each axis to change the unbalanced level of rotor. One pair of relative vibration measuring points and one pair of absolute vibration measuring points are arranged at the ends of cylinder. Signals are synchronously acquired by key phase trigger. Herein, high-pressure air with the maximum pressure of $1 \mathrm{MPa}$, provided by screw compressor, is considered as the air source of test rig.

Six seals are installed on the internal wall of the cylinder as shown in Figure 4. Negative dislocated seal is installed in the middle of the cylinder. High-pressure airflow enters from the middle seal and then flows out through the cavities of three seals at the two ends.

The stator of seal experimental piece has seven tapered straight teeth, and the rotor is smooth. The geometric parameters of the seal are shown in Figure 5, in which the dislocated rate $\gamma$ of NDS is defined as

$$
\gamma=\frac{l}{R-r}
$$

\subsection{Identification Method for Seal Rotordynamic Characteristics}

4.2.1. Equivalent Seal Force Identification Model. The airflow force inside multistage seals can be equivalent in the vertical and horizontal directions at the two ends of cylinder, as shown in Figure 6.

The cylinder is regarded as a linear system under microamplitude excitation. Regarding the vibration coupling in vertical and horizontal directions, the relationship between horizontal and vertical vibrations at the ends of the cylinder is expressed as

$$
\begin{aligned}
\left(\begin{array}{c}
\vec{F}_{\vec{F}^{x 1}} \\
\vec{F}_{y 1}^{x 2} \\
\vec{F}_{y 2}
\end{array}\right) & =\left(\begin{array}{llll}
\vec{Z}_{11} & \vec{Z}_{12} & \vec{Z}_{13} & \vec{Z}_{14} \\
\vec{Z}_{21} & \vec{Z}_{22} & \vec{Z}_{23} & \vec{Z}_{24} \\
\vec{Z}_{31} & \vec{Z}_{32} & \vec{Z}_{33} & \vec{Z}_{34} \\
\vec{Z}_{41} & \vec{Z}_{42} & \vec{Z}_{43} & \vec{Z}_{44}
\end{array}\right)\left(\begin{array}{c}
\vec{X}_{1} \\
\vec{X}_{2} \\
\vec{Y}_{2}
\end{array}\right), \\
\vec{Z} & =\left(\begin{array}{llll}
\vec{Z}_{11} & \vec{Z}_{12} & \vec{Z}_{13} & \vec{Z}_{14} \\
\vec{Z}_{21} & \vec{Z}_{22} & \vec{Z}_{23} & \vec{Z}_{24} \\
\vec{Z}_{31} & \vec{Z}_{32} & \vec{Z}_{33} & \vec{Z}_{34} \\
\vec{Z}_{41} & \vec{Z}_{42} & \vec{Z}_{43} & \vec{Z}_{44}
\end{array}\right),
\end{aligned}
$$

where $X$ and $Y$ represent the absolute vibration of cylinder in horizontal and vertical directions, respectively, and $\vec{Z}$ is the matrix of cylinder impedance function related to the structural sizes of cylinder, the stiffness of support spring, and the frequency of excitation, which can be obtained in 


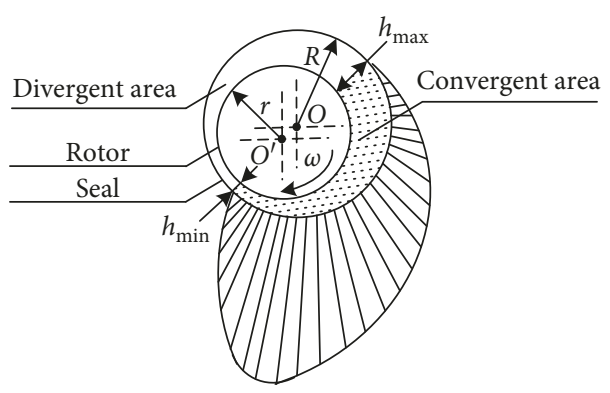

(a)

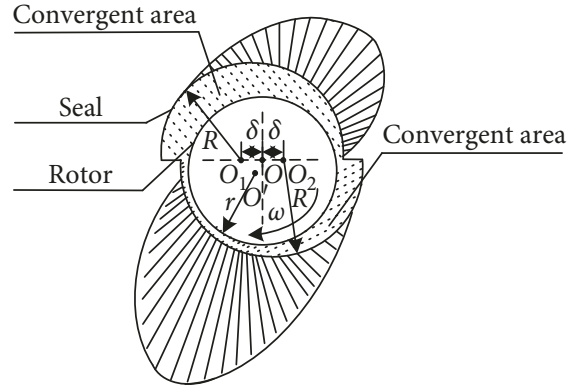

(b)

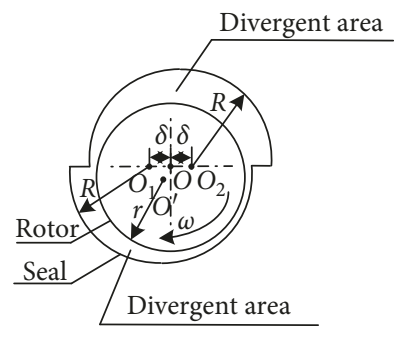

(c)

Figure 1: Schematics of the hydrodynamic pressure effects of cylinder seal and dislocated seals. (a) Cylinder seal. (b) Positive dislocated seal. (c) Negative dislocated seal.

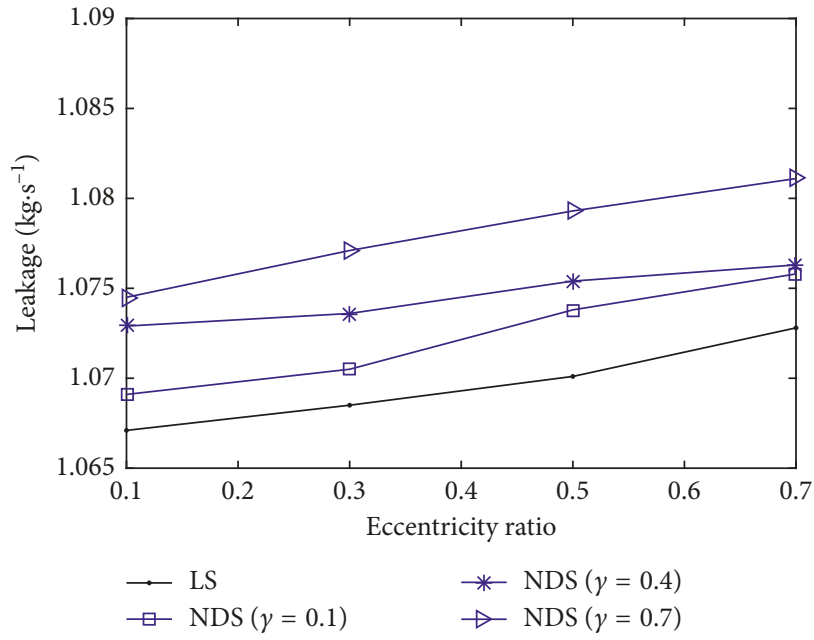

Figure 2: Effect of eccentricity on seal leakage characteristics.

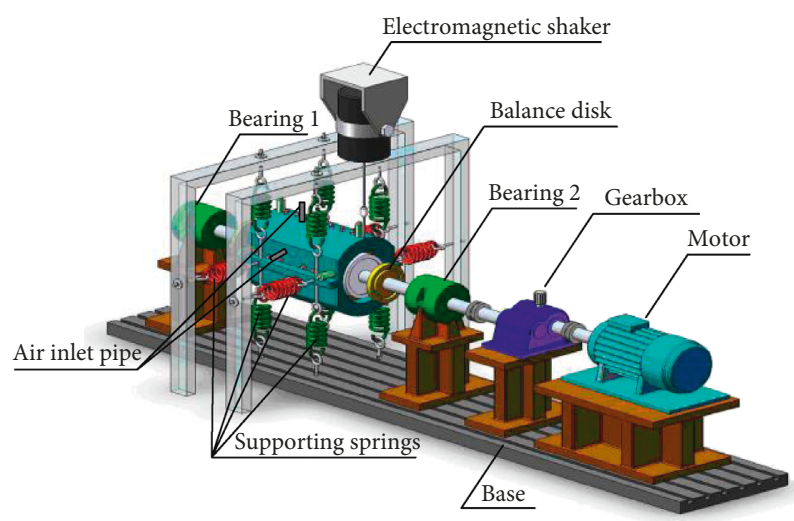

Figure 3: Schematic diagram of seal test rig.

respect of the excitation experiments of four points in vertical and horizontal directions at the ends of cylinder. The exciting points are revealed in Figure 7.

4.2.2. Rotordynamic Coefficients Identification. Considering the effect of cylinder deflection, the total horizontal and vertical airflow forces of the cylinder are, respectively, synthesized from the two ends of air force, i.e.,

$$
\left\{\begin{array}{l}
\vec{F}_{x}=\vec{F}_{x_{1}}+\vec{F}_{x_{2}}, \\
\vec{F}_{y}=\vec{F}_{y_{1}}+\vec{F}_{y_{2}} .
\end{array}\right.
$$

The subscripts 1 and 2 of the variable $x$ indicate the left and the right ends of the cylinder, respectively.

The kinetic equation of seal-rotor system can be structured as

$$
-\left[\begin{array}{l}
\vec{F}_{x} \\
\vec{F}_{y}
\end{array}\right]=\left[\begin{array}{ll}
H_{x x} & H_{x y} \\
H_{y x} & H_{y y}
\end{array}\right]\left[\begin{array}{l}
\vec{X}_{r} \\
\vec{Y}_{r}
\end{array}\right],
$$

where $\vec{X}_{r}$ and $\vec{Y}_{r}$ are the relative vibration between cylinder and rotor; $\vec{F}_{x}$ and $\vec{F}_{y}$ are joint air forces in the horizontal and vertical directions of cylinder, respectively; $H_{i j}=k_{i j}+i \omega c_{i j}$, in which $k_{i j}$ and $c_{i j}$ are seal stiffness and damping coefficients, respectively; the subscript $x x$ and $y y$ denote direct coefficients and $x y$ and $y x$ indicate cross coefficients; $\omega$ represents excitation frequency. In this study, the cylinder is only affected by airflow force with the same frequency. In this case, $\omega$ can be regarded as rotational frequency. Thus, equation (5) can be written as

$$
-\left[\begin{array}{c}
\vec{F}_{x} \\
\vec{F}_{y}
\end{array}\right]=\left[\begin{array}{cccc}
\vec{X}_{r} & \vec{Y}_{r} & 0 & 0 \\
0 & 0 & \vec{X}_{r} & \vec{Y}_{r}
\end{array}\right]\left[\begin{array}{l}
H_{x x} \\
H_{x y} \\
H_{y x} \\
H_{y y}
\end{array}\right] .
$$

Here, equation (6) contains 2 equations and 4 unknowns. To solve this problem, 4 independent equations must be constructed. Therefore, changing the unbalance level of the rotor to obtain two groups of forces and relative vibrations and then build 4 independent equations in two unbalanced states as one matrix, i.e.,

$$
-\left[\begin{array}{c}
\vec{F}_{x, \mathrm{I}} \\
\vec{F}_{y, \mathrm{I}} \\
\vec{F}_{x, \mathrm{II}} \\
\vec{F}_{y, \mathrm{II}}
\end{array}\right]=\left[\begin{array}{cccc}
\vec{X}_{r, \mathrm{I}} & \vec{Y}_{r, \mathrm{I}} & 0 & 0 \\
0 & 0 & \vec{X}_{r, \mathrm{I}} & \vec{Y}_{r, \mathrm{I}} \\
\vec{X}_{r, \mathrm{II}} & \vec{Y}_{r, \mathrm{II}} & 0 & 0 \\
0 & 0 & \vec{X}_{r, \mathrm{II}} & \vec{Y}_{r, \mathrm{II}}
\end{array}\right]\left[\begin{array}{l}
H_{x x} \\
H_{x y} \\
H_{y x} \\
H_{y y}
\end{array}\right],
$$

where the subscripts I and II represent two sets of different equilibrium states. 


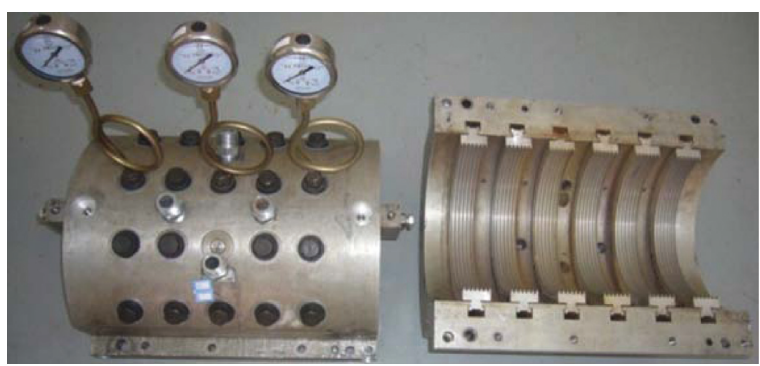

(a)

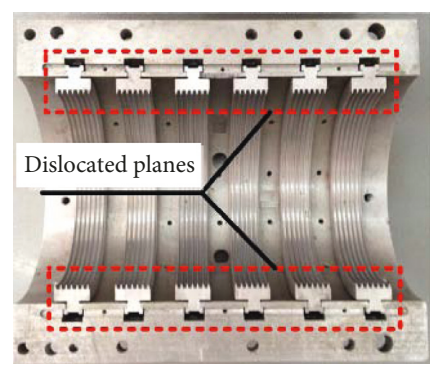

(b)

Figure 4: Cylinder and installation of NDS on seal test rig.

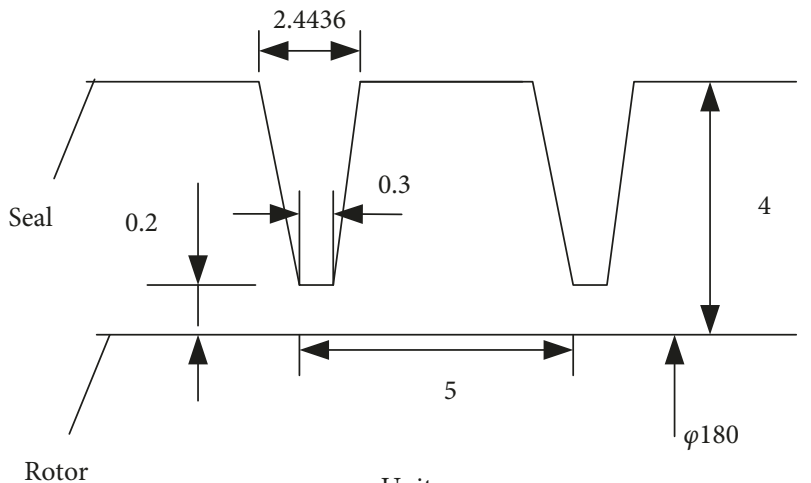

Unit: $\mathrm{mm}$

FIgURE 5: Sizes of seal.

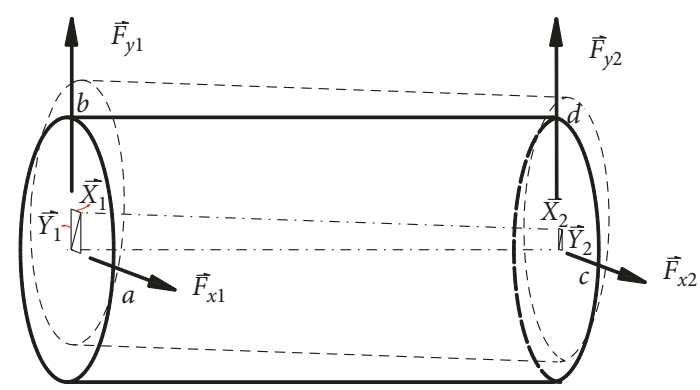

FIGURE 6: Seal force equivalent model and cylinder dynamic model.

With respect to equation (7), seal rotordynamic coefficient matrix $\left[H_{i j}\right]$ can be computed by

$$
\left[\begin{array}{l}
H_{x x} \\
H_{x y} \\
H_{y x} \\
H_{y y}
\end{array}\right]=-\left[\begin{array}{cccc}
\vec{X}_{r, \mathrm{I}} & \vec{Y}_{r, \mathrm{I}} & 0 & 0 \\
0 & 0 & \vec{X}_{r, \mathrm{I}} & \vec{Y}_{r, \mathrm{I}} \\
\vec{X}_{r, \mathrm{II}} & \vec{Y}_{r, \mathrm{II}} & 0 & 0 \\
0 & 0 & \vec{X}_{r, \mathrm{II}} & \vec{Y}_{r, \mathrm{II}}
\end{array}\right]^{-1}\left[\begin{array}{c}
\vec{F}_{x, \mathrm{I}} \\
\vec{F}_{y, \mathrm{I}} \\
\vec{F}_{x, \mathrm{II}} \\
\vec{F}_{y, \mathrm{II}}
\end{array}\right] .
$$

It is illustrated in equation (8) that the rotordynamic coefficient matrix $\left[H_{i j}\right]$ is determined by the measured equivalent airflow force and relative vibration in vertical/ horizontal direction at two ends of cylinder under two equilibrium states. The real and imaginary parts of $H_{i j}$

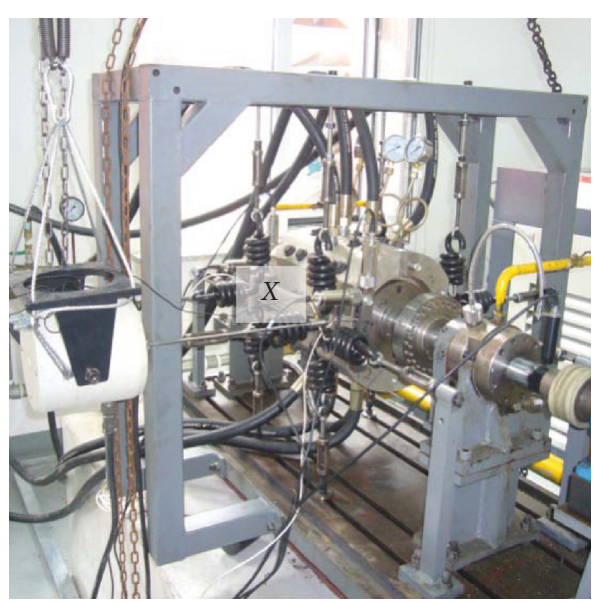

(a)

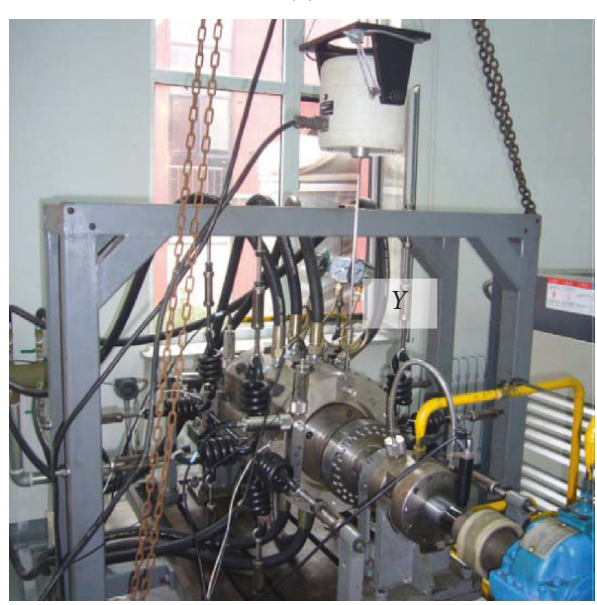

(b)

FIGURE 7: Shaker excitation experiments. (a) Shaker excited in $x$ direction. (b) Shaker excited in $y$ direction.

are stiffness coefficient $k_{i j}$ and damping coefficient $c_{i j}$, respectively.

\section{Experiment of Seal-Rotor System Stability}

5.1. Test Rig for Measuring Seal-Rotor System Stability. The test rig of seal-rotor system is shown in Figure 8. Figure 9 shows the current signal diagram of electromagnetic bearing 


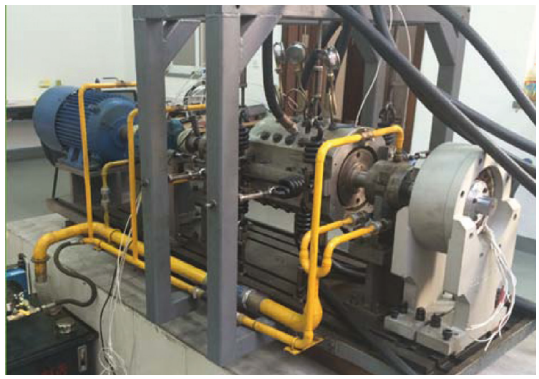

(a)

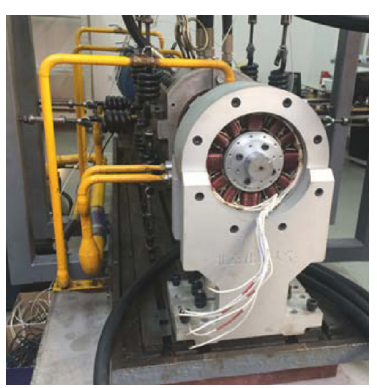

(b)

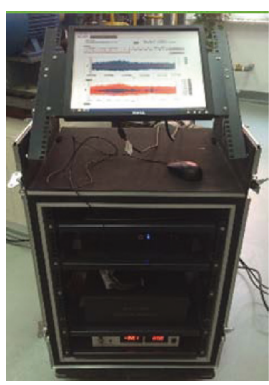

(c)

FIGURE 8: Rotor stability test rig. (a) The sealing performance testing platform for electromagnetic bearing exciter is installed. (b) Electromagnetic bearing exciter. (c) Several mining and control systems.
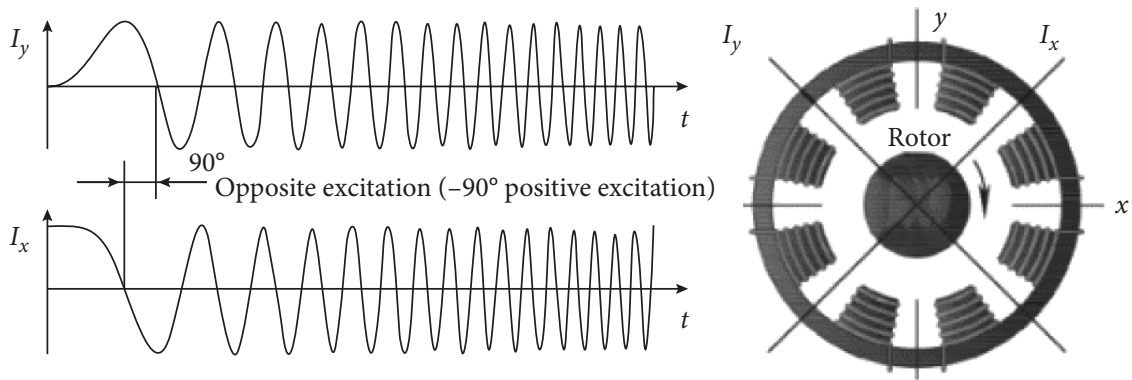

FIgURE 9: Current signal of electromagnetic bearing exciter.

exciter. The excitation coil current is harmonic signal expressed by

$$
\left\{\begin{array}{l}
I_{x}(t)=a A_{0} \cos \omega t \\
I_{y}(t)=b A_{0} \cos \omega t
\end{array}\right.
$$

where $I_{x}$ and $I_{y}$ are the currents in $x$ and $y$ directions, respectively; $A_{0}$ is the amplitude of current signal; $a$ and $b$ are the coefficients of adjusting $A_{0}$ in $x$ and $y$ directions, respectively.

\subsection{Identification Method of Seal-Rotor System Stability}

5.2.1. Dynamic Model of Rotor-Seal System. The rotor model of multiple-degree-of-freedom (MDoF) system can be described as [34]

$$
\left(\mathbf{M}_{\mathrm{s}}+\mathbf{M}_{\mathrm{m}}\right) \ddot{\mathbf{q}}+\left[\mathbf{C}_{\mathrm{b}}-\Omega\left(\mathbf{G}_{\mathrm{s}}+\mathbf{G}_{\mathrm{m}}\right)\right] \dot{\mathbf{q}}+\left(\mathbf{K}_{\mathrm{s}}+\mathbf{K}_{\mathrm{b}}+\mathbf{K}_{\mathrm{c}}\right) \mathbf{q}=\mathbf{f}(\mathrm{t}),
$$

where $\mathbf{M}_{\mathrm{s}}$ is the mass matrix of shaft; $\mathbf{M}_{\mathrm{m}}$ indicates the mass matrix of disk; $\mathbf{C}_{\mathrm{b}}$ denotes the damping matrix of seal; $\mathbf{G}_{\mathrm{s}}$ expresses the gyroscope effect matrix of shaft; $\mathbf{G}_{\mathrm{m}}$ reveals the gyroscope effect matrix of disk; $\mathbf{K}_{\mathrm{s}}$ and $\mathbf{K}_{\mathrm{b}}$ are the stiffness matrixes of shaft and disk; $\mathbf{K}_{\mathbf{c}}$ represents the coupling stiffness matrix of shaft and disk; $\Omega$ is the angular velocity; $\mathbf{f}(t)$ is the excitation force vector which might be external and induced unbalance mass of disk, as well as a function of the rotor displacement and velocity; $\mathbf{q}$ denotes the displacement vector of both translation and rotation which has 4 degrees of freedoms $(\mu, \nu, \alpha$, and $\beta)$.
As for the MDoF rotor system in Figure 10, the effect of $\alpha$ and $\beta$ in $\mathbf{q}$ on modal recognition is not temporarily measured owing to the inability. When angular frequency is determined, equation (10) can be simplied as

$$
\mathbf{M q}+(\Omega \mathbf{G}+\mathbf{C}) \dot{\mathbf{q}}+\mathbf{K q}=\mathbf{f}(\mathrm{t})
$$

where $\mathbf{q}(\mathrm{t})=(x(t), y(t))^{T} ; \mathbf{f}(\mathrm{t})=\left(f_{x}(t), f_{y}(t)\right)^{T} ; x(t)$ and $y(t)$ are displacement responses in $x$ and $y$ directions, respectively; $f_{x}(t)$ and $f_{y}(t)$ are excitation forces in $x$ and $y$ directions, respectively.

In respect of Fourier transform, the matrix $\mathbf{H}(j \omega)$ of equation (11) frequency function is

$$
\left(\begin{array}{l}
X(j \omega) \\
Y(j \omega)
\end{array}\right)=\left(\begin{array}{ll}
H_{x x} & H_{x y} \\
H_{y x} & H_{y y}
\end{array}\right)\left(\begin{array}{l}
F_{x}(j \omega) \\
F_{y}(j \omega)
\end{array}\right),
$$

in which

$$
H_{p q}(j \omega)=\sum_{i=i_{B}, i_{F}} \sum_{r=1}^{S_{N}}\left(\frac{\mathbf{u} \overline{\mathbf{v}}^{T}}{j \omega-\lambda}+\frac{\overline{\mathbf{u}} \mathbf{v}^{T}}{j \omega-\bar{\lambda}}\right)_{i . r}(p, q=x, y),
$$

where $j=\sqrt{-1}$ is the unit of complex number; $\omega$ indicates angular frequency; $H_{p q}$ denotes the response function in $p$ direction excited from $q$ direction; $i_{F}$ and $i_{B}$ denote the positive and reverse precessions, respectively; $X(j \omega), Y(j \omega)$, $F_{x}(j \omega)$, and $F_{y}(j \omega)$ are the Fourier transforms of $x(t), y(t)$, $f_{x}(t)$, and $f_{y}(t)$, respectively; $S_{N}$ indicates the total number of support points; $\mathbf{u}$ is the mode vector; $\mathbf{v}$ expresses the vector structured by adjoins matrices; $\lambda$ is the eigenvalue; $\overline{\mathbf{v}}$ and $\overline{\mathbf{u}}$ are the conjugation of $\mathbf{v}$ and $\mathbf{u}$, respectively. 

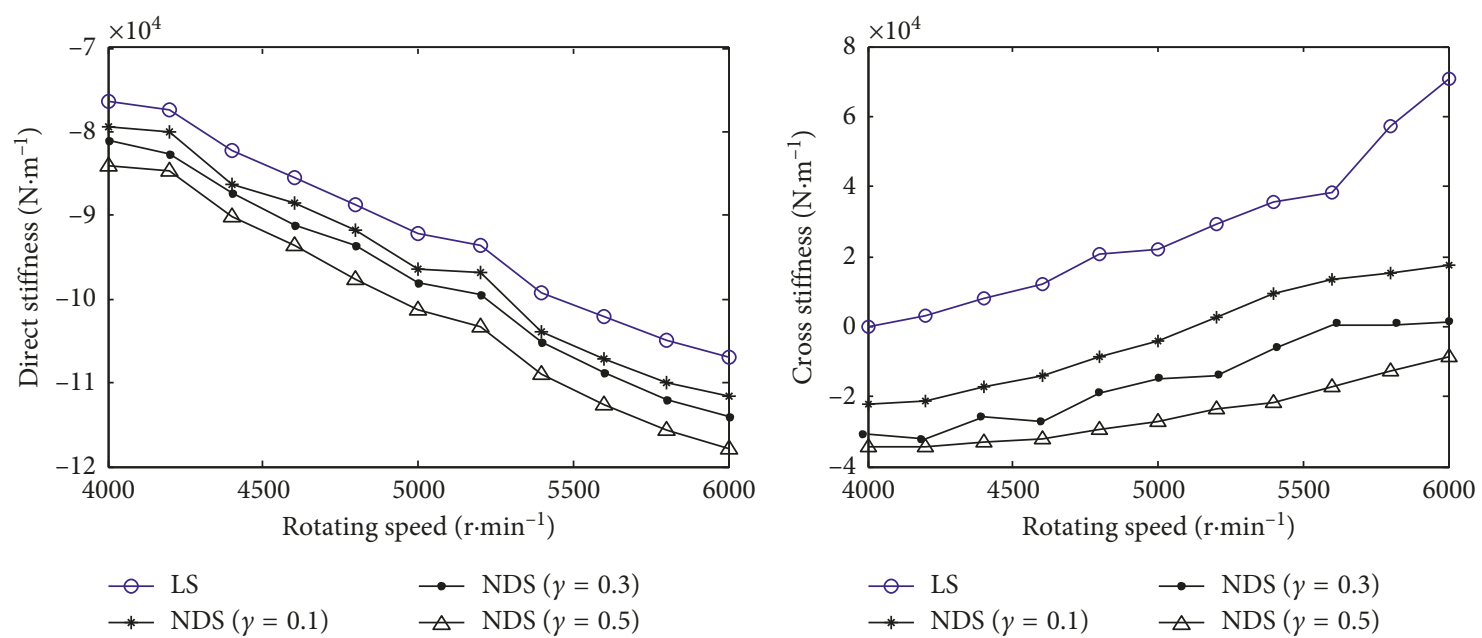

(a)
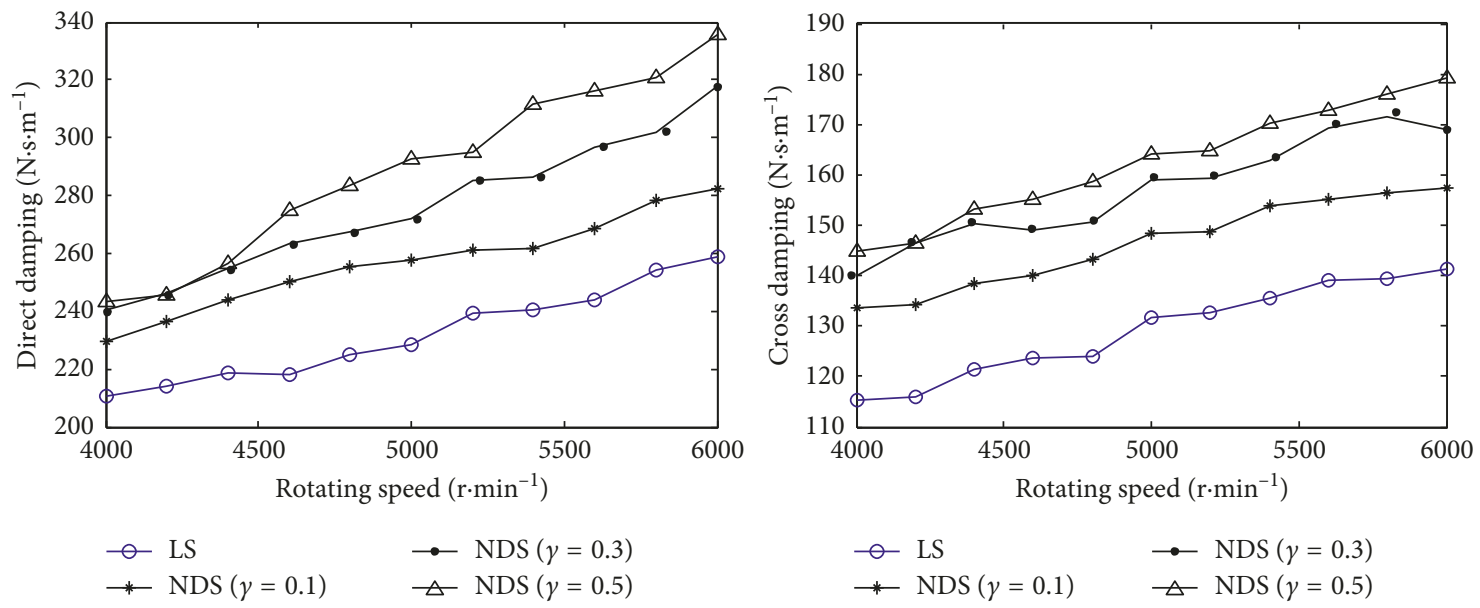

(b)

Figure 10: Dynamic characteristic coefficients as a function of rotating speed. (a) Stiffness coefficients. (b) Damping coefficients.

5.2.2. Rotor System Stability Evaluation Based on StandardLogarithmic Decrement Rate. Rotor stability is generally determined by the decrement rate of first-order $\left(1^{\text {st }}\right)$ rotor modal. The logarithmic attenuation rate of $1^{\text {st }}$ precession is obtained from the $1^{\text {st }}$ modal eigenvalue $\left(\lambda_{1 F}\right)$ of the rotor system, i.e.,

$$
\delta=\frac{-2 \pi \operatorname{Re}\left(\lambda_{1 F}\right)}{\operatorname{Im}\left(\lambda_{1 F}\right)}=\frac{2 \pi \xi}{\sqrt{1-\xi^{2}}},
$$

where $\xi$ is the damping ratio of rotor system.

\subsubsection{Analysis of Complex Field Model}

(1) Transformation Mechanism of Direct Frequency Response Function. In light of equation (12), frequency response function includes the modal parameters of rotor positive and negative precession. However, the damping ratio of firstorder forward precession plays a key role on rotor instability. It is necessary to separate positive and negative precession modes for frequency response function. Introducing complex displacement $r(t)$, the vibrational displacement signals $x(t)$ and $y(t)$ of real field are described as

$$
\begin{gathered}
\left(\begin{array}{l}
r(t) \\
\bar{r}(t)
\end{array}\right)=\left(\begin{array}{cc}
1 & j \\
1 & -j
\end{array}\right)\left(\begin{array}{l}
x(t) \\
y(t)
\end{array}\right), \\
\left(\begin{array}{l}
f_{r}(t) \\
\bar{f}_{r}(t)
\end{array}\right)=\left(\begin{array}{cc}
1 & j \\
1 & -j
\end{array}\right)\left(\begin{array}{l}
f_{x}(t) \\
f_{y}(t)
\end{array}\right),
\end{gathered}
$$

where $\bar{r}(t)$ and $\bar{f}_{r}(t)$ are the conjugate of $r(t)$ and $f_{r}(t)$.

The frequency response function matrix $\mathbf{G}(j \omega)$ in the complex field is expressed by

$$
\left(\begin{array}{l}
R(t) \\
\bar{R}(t)
\end{array}\right)=\left(\begin{array}{ll}
G_{r r} & G_{r \bar{r}} \\
G_{\bar{r} r} & G_{\overline{r r}}
\end{array}\right)\left(\begin{array}{l}
F_{r}(j \omega) \\
\bar{F}_{r}(j \omega)
\end{array}\right),
$$

where $R(j \omega)$ and $\bar{R}(j \omega)$ are the Fourier transform of $r(t)$ and $\bar{r}(t)$.

Assuming transformation matrix $\mathbf{J}=\left(\begin{array}{cc}1 & j \\ 1 & -j\end{array}\right)$, the Fourier transform of equations (15) and (16) is gained as

$$
\begin{aligned}
\left(\begin{array}{l}
R(j \omega) \\
R(j \omega)
\end{array}\right) & =\mathbf{J}\left(\begin{array}{l}
X(j \omega) \\
Y(j \omega)
\end{array}\right), \\
\left(\begin{array}{l}
F_{r}(j \omega) \\
\bar{F}_{r}(j \omega)
\end{array}\right) & =\mathbf{J}\left(\begin{array}{l}
F_{x}(j \omega) \\
F_{y}(j \omega)
\end{array}\right) .
\end{aligned}
$$


Based on equations (12)-(16), we gain

$$
\left(\begin{array}{l}
R(j \omega) \\
\bar{R}(j \omega)
\end{array}\right)=\mathbf{J}\left(\begin{array}{cc}
H_{x x} & H_{x y} \\
H_{y x} & H_{y y}
\end{array}\right) \mathbf{J}^{-1}\left(\begin{array}{c}
F_{r}(j \omega) \\
\bar{F}_{r}(j \omega)
\end{array}\right) .
$$

The relationship between $\mathbf{G}(j \omega)$ and $\mathbf{H}(j \omega)$ is obtained as

$$
\left(\begin{array}{cc}
G_{r r} & G_{r \bar{r}} \\
G_{\bar{r} r} & G_{\overline{r r}}
\end{array}\right)=\mathbf{J}\left(\begin{array}{cc}
H_{x x} & H_{x y} \\
H_{y x} & H_{y y}
\end{array}\right) \mathbf{J}^{-1},
$$

where $H_{p q}(-j \omega)=\bar{H}_{p q}(j \omega)(p, q=x, y)$ and $G_{r r}(j \omega)$ is exerted by $\mathbf{G}(-j \omega)=\mathbf{J H}(-j \omega) \mathbf{J}^{-1}$.

In terms of the above analysis, the frequency response function on negative half axis can be gained so that the frequency response functions of positive and negative precessions are separated to weaken the effect of modal superposition and realize the modal separation of positive and negative precessions.

(2) Physical Realization of Direct Frequency Response Function. In this study, electromagnet bearing is applied to exert rotating force to directly test frequency response function $\mathbf{G}(j \omega)$. When the exerted excitation forces to rotor are $f_{x}(t)=A_{x} \cos (\omega \mathrm{t})$ and $f_{y}(t)=A_{y} \cos (\omega \mathrm{t})$, the force $F_{r}(t)$ in complex number domain is

$$
F_{r}(t)=f_{x}(t)+j f_{y}(t)= \begin{cases}A e^{+j \omega t}, & A_{x}=A_{y}=A, \\ A e^{-j \omega t}, & -A_{x}=A_{y}=A .\end{cases}
$$

As shown in equation (22), the rotation direction of $F_{r}$ is positive precession direction that $F_{r}(j \omega)=0$ in negative frequency domain as $A_{x}=A_{y}$. Otherwise, the rotation direction of $F_{r}$ is the reverse precession direction when $A_{x}$ is equal to $A_{y}$ and opposite against $A_{y}$ in direction. Therefore, under the effect of rotating force exerted to the rotor system, the positive and negative precessions of rotor system can be decomposed under complex field. When positive excitation force is exerted by

$$
G_{r f}(j \omega)=\frac{R(j \omega)}{F_{r}(j \omega)}, \quad \omega \geq 0 .
$$

When reverse excitation force is applied, there is

$$
G_{\bar{r} \bar{f}}(j \omega)=\frac{\bar{R}(j \omega)}{\bar{F}_{r}(j \omega)}, \quad \omega \geq 0 .
$$

The distribution of frequency response function $G_{r f}(j \omega)$ on positive and negative axis can be obtained in terms of the formula $G_{r f}(-j \omega)=\bar{G}_{\bar{r} \bar{f}}(j \omega)$ [34].

\section{Experimental Results and Discussion}

\subsection{Experimental Results of Seal Rotordynamic Characteristics}

6.1.1. Influence of Rotational Speed on Seal Rotordynamic Characteristics. Based on the experiment, the relationship between seal rotordynamic coefficients and rotational speeds under different dislocated rates is shown in Figure 10 when inlet/outlet pressure ratio is 6 .
As presented in Figure 10, direct stiffness coefficient decreases while both cross-stiffness coefficient and damping coefficient increase, as rotational speed increases. Comparing with the traditional cylindrical seal, the cross stiffness decreases and the direct damping for the NDS increases. The negative dislocated structure can effectively reduce seal hydrodynamic pressure effect, seal tangential force, and cross-stiffness coefficient. The dislocated structure increases flow resistance and increases the direct damping coefficient of the NDS.

6.1.2. Influence of Inlet/Outlet Pressure Ratio on Seal Rotordynamic Characteristics. Figure 11 shows the relationship between seal rotordynamic coefficients and inlet/ outlet pressure ratios under different dislocated rates. The rotational speed is $6000 \mathrm{r} / \mathrm{min}$. As illustrated in Figure 11, with the increasing inlet/outlet pressure ratio, the direct stiffness coefficient decreases while the cross-stiffness coefficient and damping coefficient increase. Compared with the traditional cylindrical seal, the cross stiffness of NDS obviously reduces and its direct damping obviously increases. Besides, with the increase of dislocated ratio, the effect of inlet/outlet pressure ratio on the seal rotordynamic coefficient is more obvious. Therefore, negative dislocated structure can effectively reduce pressure difference and cross-stiffness coefficient, increase direct damping coefficient and improve seal stability as well.

6.2. Experimental Results of Seal Stability. Figures 12 and 13 display the effects of rotating speed and inlet/outlet pressure ratio on the logarithmic decrement rate.

In Figures 12 and 13, we can find that the increase of speed and inlet/outlet pressure ratio decreases the logarithmic decrement rate. Under the same service condition, the logarithmic decrement rate of the NDS is larger than that of the traditional cylindrical seal. Besides, the increase of dislocated rate enlarges the logarithmic decrement rate. The NDS has the capacity of improving rotor stability by effectively reducing seal hydrodynamic pressure effect and increasing flow resistance. Therefore, the developed NDS can effectively improve rotor system stability in turbomachinery.

\section{Conclusions}

The aim of this paper is to develop a new negative dislocated seal (NDS) and study the effects of dislocated rate, rotating speed, inlet/outlet pressure ratio on seal rotordynamic coefficients, and rotor stability from an experimental perspective. Some conclusions are summarized below.

(1) The rotordynamic characteristics of both the NDS and the traditional cylindrical seal are experimentally investigated by the unbalanced same frequency excitation method. From this study, we find that with the increase of rotational speed and inlet/outlet pressure ratio, the seal direct stiffness coefficient decreases while the cross-stiffness coefficient and 

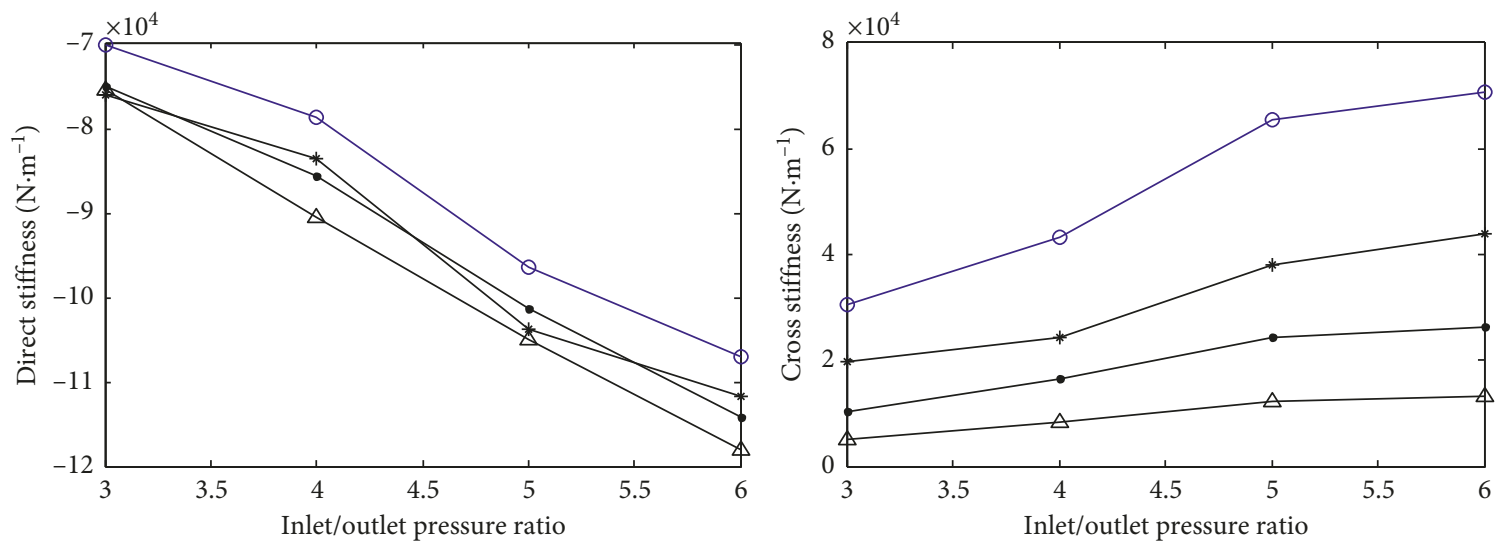

$$
\begin{array}{ll}
\multimap \text { LS } & \rightarrow \operatorname{NDS}(\gamma=0.3) \\
* \operatorname{NDS}(\gamma=0.1) & \triangle \operatorname{NDS}(\gamma=0.5)
\end{array}
$$

$$
\begin{array}{ll}
\multimap \text { LS } & \rightarrow \operatorname{NDS}(\gamma=0.3) \\
\rightarrow \text { NDS }(\gamma=0.1) & \triangle \operatorname{NDS}(\gamma=0.5)
\end{array}
$$

(a)
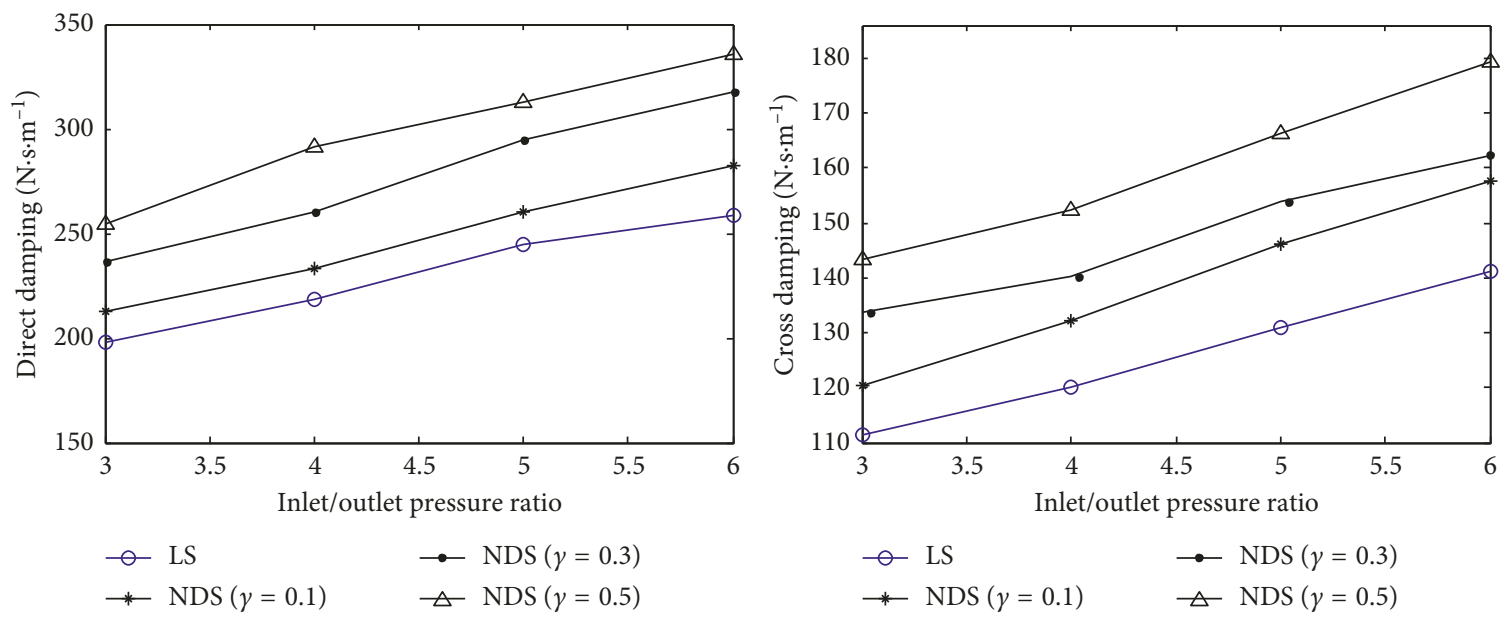

(b)

FIGURE 11: Dynamic characteristics coefficients as a function of inlet/out pressure ratio. (a) Stiffness coefficients. (b) Damping coefficients.

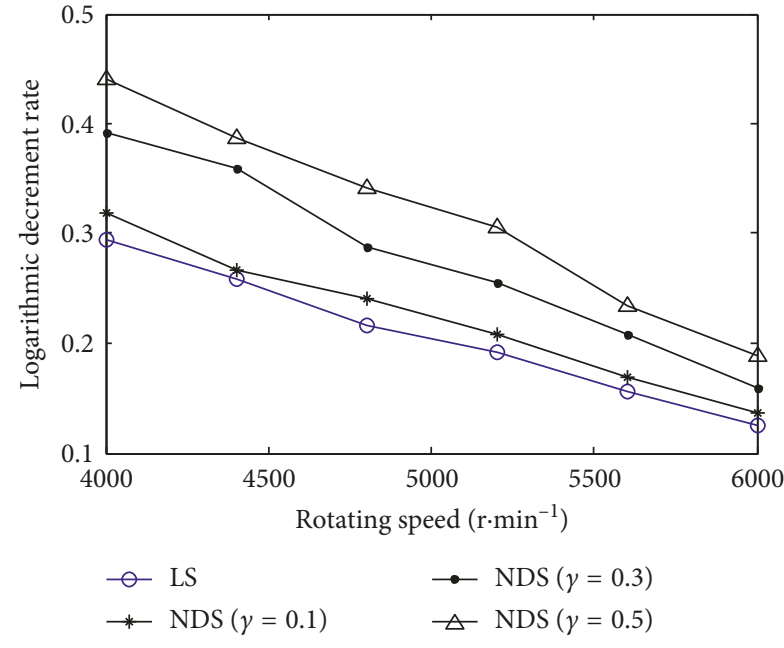

FIGURE 12: Influence of rotating speed on logarithmic decrement rate.

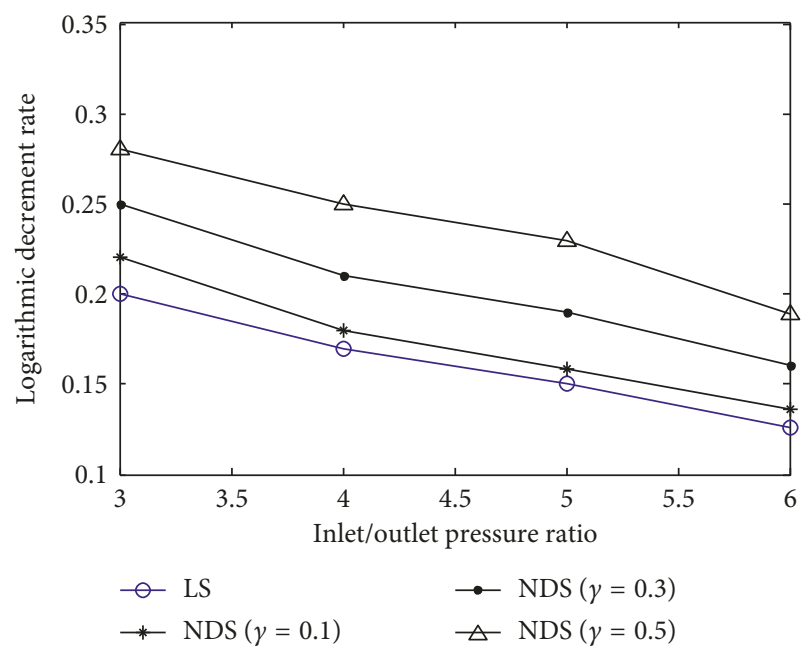

FIGURE 13: Influence of inlet/out pressure ratios on logarithmic decrement rate. 
damping coefficient increase. Comparing with the traditional cylindrical seal, the NDS can reduce the cross-stiffness coefficient and improve the direct damping coefficient.

(2) The rotor stability of both the new negative dislocated seal and the traditional cylindrical seal is studied from an experiment perspective, by adopting the rotor stability identification method based on an electromagnetic bearing exciter. The results reveal that the logarithmic decrement rate of seal rotor system decreases when the rotational speed and inlet/outlet pressure ratio increase. Additionally, the NDS improves the logarithmic decrement rate of the seal rotor system and rotor stability relative to the traditional cylindrical seal.

The goal of this study is to develop a valuable seal structure design (NDS) and deeply investigate seal rotordynamic characteristics and rotor stability through experiments. The efforts of this study demonstrate that the NDS can inhibit the excitation force of seal airflow, contribute to the stability of rotor system, and improve the performance of rotating machinery.

\section{Data Availability}

The data used to support the findings of this study are included within the article.

\section{Conflicts of Interest}

The authors declare that there are no conflicts of interests regarding the publication of this article.

\section{Acknowledgments}

This paper was supported by the National Natural Science Foundation (Grant Nos. 51675351, 51605016, and 51475025), Innovative Talents Support Program of Liaoning Province for the Institutions of Higher Education (Grant No. LR2016033), and Research Start-up Funding of Fudan University.

\section{References}

[1] A. J. Smalley, M. Camatti, D. W. Childs, J. R. Hollingsworth, G. Vannini, and J. J. Carter, "Dynamic characteristics of the diverging taper honeycomb-stator seal," Journal of Turbomachinery, vol. 128, no. 4, pp. 717-724, 2006.

[2] C.-W. Fei, Y.-S. Choy, D.-Y. Hu, G.-C. Bai, and W.-Z. Tang, "Dynamic probabilistic design approach of high-pressure turbine blade-tip radial running clearance," Nonlinear Dynamics, vol. 86, no. 1, pp. 205-223, 2016.

[3] X. Yan, K. He, J. Li, and Z. Feng, "Numerical investigations on rotordynamic characteristic of hole-pattern seals with two different hole-diameters," Journal of Turbomachinery, vol. 137, no. 7, article 071011, 2015.

[4] A. Muszynska, W. D. Franklin, and D. E. Bently, "Rotor active anti-swiril control," Journal of Vibration Acoustics Stress and Reliability in Design, vol. 110, no. 2, pp. 143-150, 1988.

[5] K. K. Nielsen, D. W. Childs, and C. M. Myllerup, "Experimental and theoretical comparison of two swirl brake designs," Journal of Turbomachinery, vol. 123, no. 2, pp. 353-358, 2001.

[6] D. Sun, S. Wang, and C. W. Fei, "Numerical and experimental investigation on the effect of swirl brakes on the labyrinth seals," Journal of Engineering for Gas Turbines and Power, vol. 138, no. 3, article 032507, 2015.

[7] N. Kim, S.-Y. Park, and D. L. Rhode, "Predicted effects of shunt injection on the rotordynamics of gas labyrinth seals," Journal of Engineering for Gas Turbines and Power, vol. 125, no. 1, pp. 167-174, 2003.

[8] D. Sun, S. Wang, Y. Ai, H. Zhou, and K. M. Wang, "Experimental investigation of rotordynamic coefficients for the labyrinth seals with and without shunt injection," Journal of Vibroengineering, vol. 17, no. 8, pp. 4289-4300, 2015.

[9] D. W. Childs, J. E. Mcean Jr., M. Zhang, and S. P. Arthur, "Rotordynamic performance of a negative-swirl brake for a tooth-on-stator labyrinth," Journal of Engineering for Gas Turbines and Power, vol. 138, no. 6, article 062505, 2015.

[10] S. Y. Park, Computational Investigation of Fluid Injection Applications for the Gas Labyrinth Seal, Texas A\&M University, College Station, TX, USA, 2002.

[11] E. A. Soto and D. W. Childs, "Experimental rotordynamic coefficient results for (a) a labyrinth seal with and without shunt injection and (b) a honeycomb seal," Journal of Engineering for Gas Turbines and Power, vol. 121, no. 1, pp. 153159, 1999.

[12] P. D. Brown and D. W. Childs, "Measurement versus predictions of rotordynamic coefficients of a hole-pattern gas seal with negative preswirl," Journal of Engineering for Gas Turbines and Power, vol. 134, no. 12, article 122503, 2012.

[13] G. Von Pragenau, "Damping seals for turbomachinery," NASA Technical Paper No. 1987, NASA, Washington, DC, USA, 1982.

[14] V. Schramm, K. Willenborg, S. Kim, and S. Wittig, "Influence of a honeycomb facing on the flow through a stepped labyrinth seal," Journal of Engineering for Gas Turbines and Power, vol. 124, no. 1, pp. 140-146, 2002.

[15] D. Sun, N.-N. Liu, C.-W. Fei, G.-Y. Hu, Y.-T. Ai, and Y.-S. Choy, "Theoretical and numerical investigation on the leakage characteristics of brush seals based on fluid-structure interaction," Aerospace Science and Technology, vol. 58, pp. 207-216, 2016.

[16] D. Sun, X. Wang, C. Fei, S. Wang, and Y. Ai, "A novel negative dislocated seal and influential parameter analyses of static/ rotordynamic characteristics," Journal of Mechanical Science and Technology, vol. 32, no. 9, pp. 4125-4134, 2018.

[17] G. Vannini, S. Cioncolini, G. D. Vescovo, and M. Rovini, "Labyrinth seal and pocket damper seal high pressure rotordynamic test data," Volume 7B: Structures and Dynamics, vol. 136, no. 2, article 022501, 2014.

[18] N. Bachschmid, P. Pennacchi, and A. Vania, "Steam-whirl analysis in a high pressure cylinder of a turbo generator," Mechanical Systems and Signal Processing, vol. 22, no. 1, pp. 121-132, 2008.

[19] B. Ertas, A. Gamal, and J. Vance, "Rotordynamic force coefficients of pocket damper seals," Journal of Turbomachinery, vol. 128, no. 4, pp. 725-737, 2006.

[20] K. Kwanka, W. Ortinger, and J. Steckel, "Calculation and measurement of the influence of flow parameters on rotordynamic coefficients in labyrinth seals," in Proceedings of a Workshop on Rotordynamic Instability Problems in High Performance Turbomachinery, pp. 121-129, Texas A\&M University, College Station, TX, USA, June 1993. 
[21] R. Tiwari, S. Manikandan, and S. K. Duivedy, "A review of the experimental estimation of the rotor dynamic parameters of seals," The Shock and Vibration Digest, vol. 37, no. 4, pp. 261-284, 2005.

[22] T. B. Sprowl and D. W. Childs, "A study of the effects of inlet preswirl on the dynamic coefficients of a straight-bore honeycomb gas damper seal," Journal of Engineering for Gas Turbines and Power, vol. 129, no. 1, pp. 220-229, 2007.

[23] A. Picardo and D. W. Childs, "Rotordynamic coefficients for a tooth-on-stator labyrinth seal at 70 bar supply pressures: measurements versus theory and comparisons to a holepattern stator seal," Journal of Engineering for Gas Turbines and Power, vol. 127, no. 4, pp. 843-855, 2005.

[24] D. Childs and K. Hale, "A test apparatus and facility to identify the rotordynamic coefficients of high-speed hydrostatic bearings," Journal of Tribology, vol. 116, no. 2, pp. 337-343, 1994.

[25] D. W. Childs and A. J. Gansle, "Experimental leakage and rotordynamic results for helically grooved annular gas seals," Journal of Engineering for Gas Turbines and Power, vol. 118, no. 2, pp. 389-393, 1996.

[26] D. W. Childs, L. E. Rodriguez, V. Cullotta, A. Al-Ghasem, and M. Graviss, "Rotordynamic-coefficients and static (equilibrium loci and leakage) characteristics for short, laminar-flow annular seals," Journal of Tribology, vol. 128, no. 2, pp. 378387, 2006.

[27] Q. H. Li, W. M. Wang, L. F. Chen, and D. Sun, "Rotor-system log-decrement identification using short-time fouriertransform filter," International Journal of Rotating Machinery, vol. 2015, Article ID 809785, 12 pages, 2015.

[28] B. C. Pettinato, C. H. Cloud, and R. S. Campos, "Shop acceptance testing of compressor rotordynamic stability and theoretical correlation," in Proceedings of the 39th Turbomachinery Symposium, pp. 31-42, Houston, TX, USA, October 2010.

[29] P. Zhong, Rotor Bearing System Identification Using Time Domain Method, University of Virginia, Charlottesville, VA, USA, 1997.

[30] C. H. Cloud, Stability of Rotors Supported by Tilting Pad Journal Bearings, University of Virginia, Charlottesville, VA, USA, 2007.

[31] C.-Y. Joh and C.-W. Lee, "Use of dFRFS for diagnosis of asymmetric/anisotropic properties in rotor-bearing system," Journal of Vibration and Acoustics, vol. 118, no. 1, pp. 64-69, 1996.

[32] C.-W. Lee, "A complex modal testing theory for rotating machinery," Mechanical Systems and Signal Processing, vol. 5, no. 2, pp. 119-137, 1991.

[33] N. Takahashi, Y. Magara, M. Narita, and H. Miura, "Rotordynamic evaluation of centrifugal compressor using electromagnetic exciter," Journal of Engineering for Gas Turbines and Power, vol. 134, no. 3, article 032505, 2012.

[34] W. Wang, Q. Li, J. Gao, J. Yao, and P. Allaire, "An identification method for damping ratio in rotor systems," $\mathrm{Me}$ chanical Systems and Signal Processing, vol. 68-69, pp. 536-554, 2016.

[35] D. W. Childs, M. Graviss, and L. E. Rodriguez, "Influence of groove size on the static and rotordynamic characteristics of short, laminar-flow annular seals," Journal of Tribology, vol. 129, no. 2, pp. 398-406, 2007. 


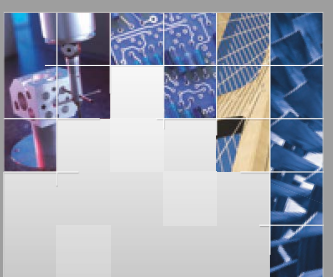

\section{Enfincering}
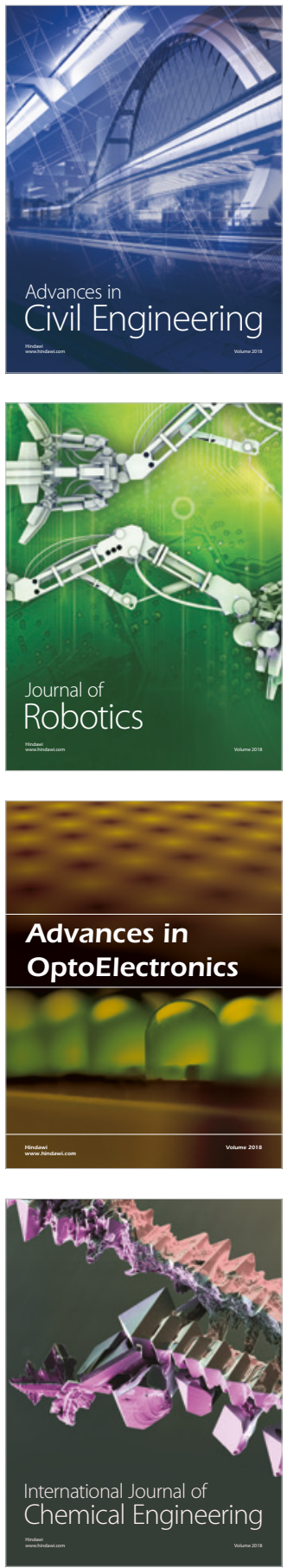

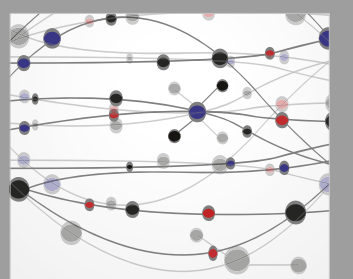

\section{Rotating \\ Machinery}

The Scientific World Journal

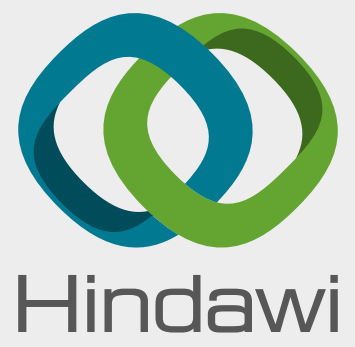

Submit your manuscripts at

www.hindawi.com
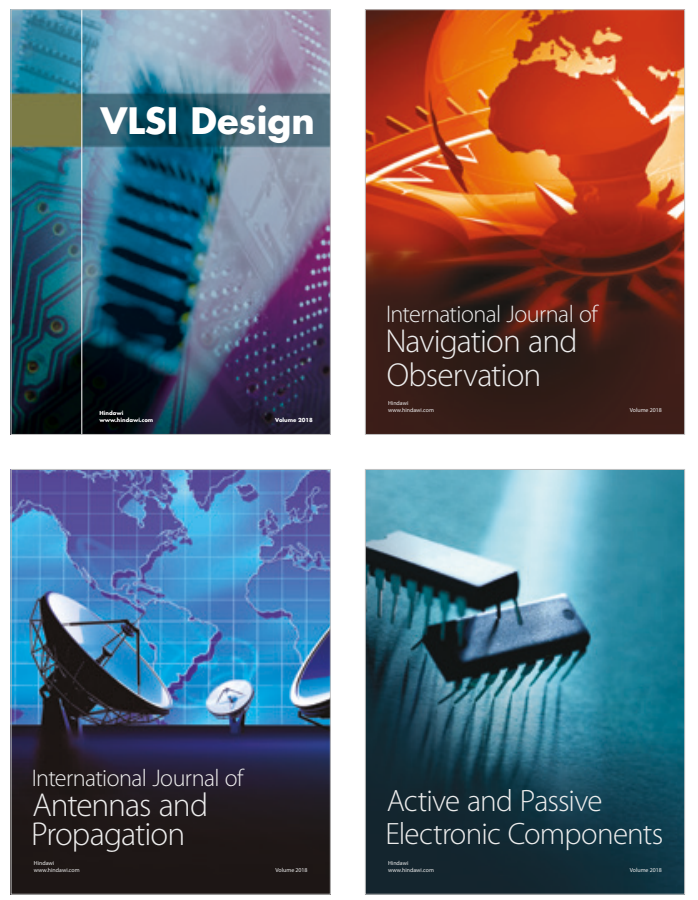
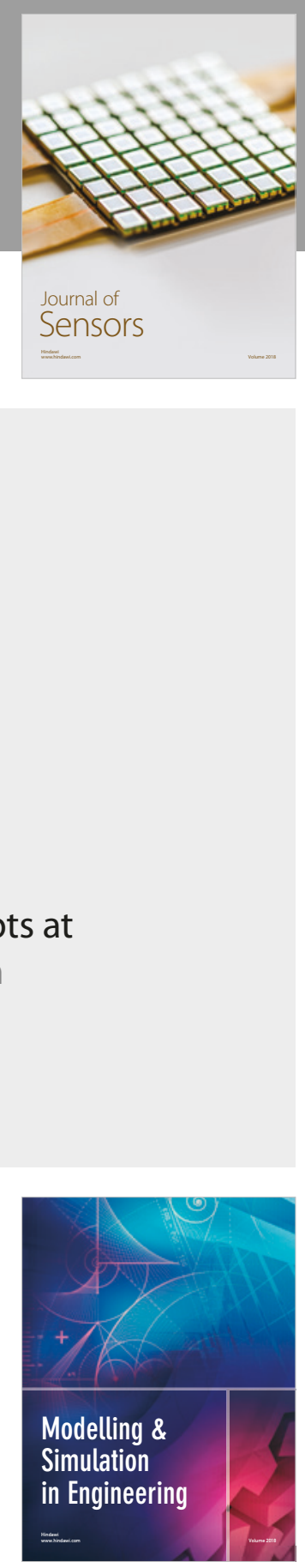

\section{Advances \\ Multimedia}
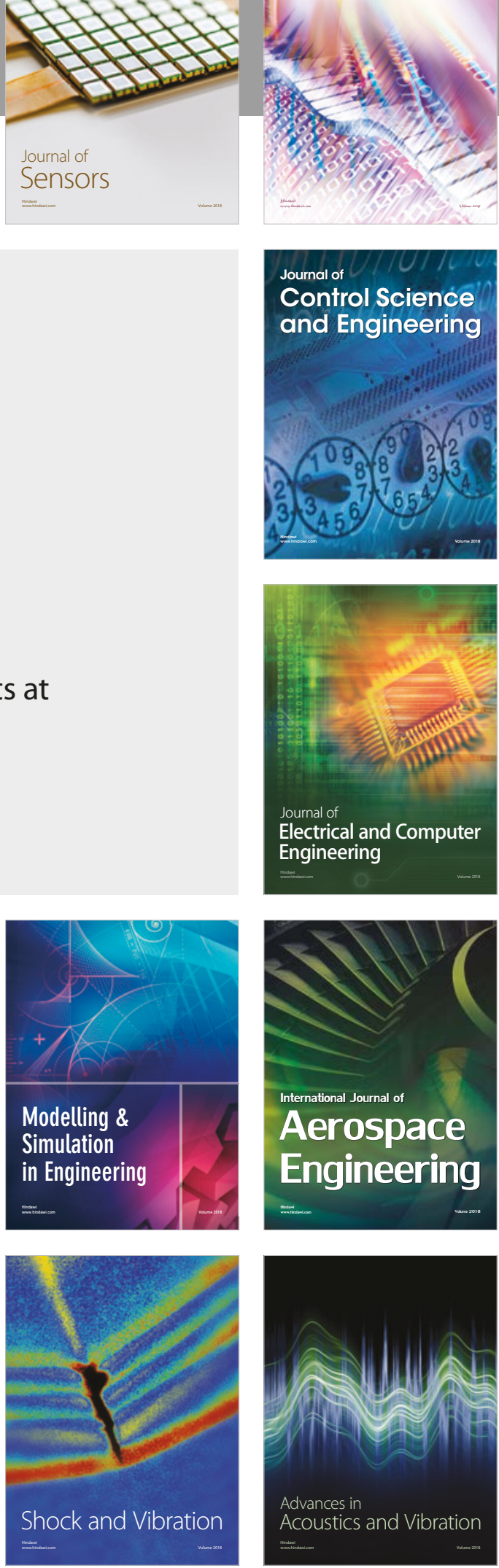